\title{
MALP-2, an agonist of TLR6, promotes the immune status without affecting the differentiation capacity of umbilical cord mesenchymal stem cells
}

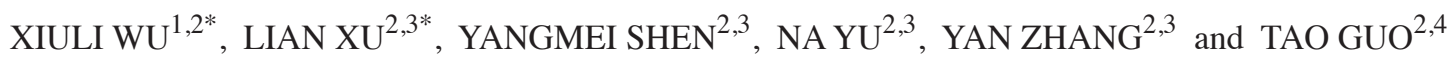 \\ ${ }^{1}$ Department of Laboratory Medicine, West China Second Hospital, Sichuan University, Chengdu, Sichuan 610041; \\ ${ }^{2}$ Key Laboratory of Birth Defects and Related Diseases of Women and Children, Sichuan University, \\ Ministry of Education, Beijing 100816; Departments of ${ }^{3}$ Pathology and ${ }^{4}$ Gynecology and Obstetrics, \\ West China Second Hospital, Sichuan University, Chengdu, Sichuan 610041, P.R. China
}

Received July 5, 2016; Accepted April 7, 2017

DOI: $10.3892 /$ etm.2017.5262

\begin{abstract}
Mesenchymal stem cells (MSCs) are increasingly used in cell-based therapy due to their multiple differentiation capacity, low expression of co-stimulatory factors and immunosuppressive effect. However, accumulating studies reported the recognition and rejection of engrafted MSCs, which eventually led to the fail of clinical trials. Toll-like receptors (TLRs) are important in mediating the immune response. In the present study, macrophage-activated lipopeptide-2 (MALP-2) was introduced to activate the TLR6 pathway in umbilical cord MSCs (UCMSCs). PBLs isolated from healthy volunteers were co-cultured with UCMSCs to measure whether activation of TLR6 of UCMSCs could stimulate immune responses. Reverse transcription-quantitative polymerase chain reaction and immunohistochemistry were performed to detect pro-inflammatory molecules and differentiation status of UCMSCs, respectively. The results indicated that activation of TLR6 in UCMSCs increased the proliferation of peripheral blood leukocytes (PBLs) and enhanced the release of lactate dehydrogenase in damaged UCMSCs, which confirmed the role of TLR6 in promoting the immunogenicity of UCMSCs. Furthermore, quantitative polymerase chain reaction demonstrated that the expression of proinflammatory molecules (including IL-1 $\beta$, IL-6, IL-8, IL-10, CCL1 and CCL4) was induced, whereas the expression of stem cell markers (K1f4 and Nanog) was inhibited. The differentiation results indicated
\end{abstract}

Correspondence to: Dr Tao Guo, Department of Gynecology and Obstetrics, West China Second Hospital, Sichuan University, 24 South People Road, Chengdu, Sichuan 610041, P.R. China

E-mail: ivan.gt@163.com

*Contributed equally

Key words: umbilical cord mesenchymal stem cells, Toll-like receptor 6, macrophage-activated lipopeptide-2, immunogenicity, biological function that activation of TLR6 had no effect on the differentiation capacity of UCMSCs. All these findings suggest that stimulation of TLR6 pathway may increase the immunogenicity of UCMSCs in in vitro detections. In conclusion, the results of the current study indicated a new role of TLR6 in regulating the biological function of UCMSCs.

\section{Introduction}

Mesenchymal stem cells (MSCs) are currently widely used in stem cell therapy for chronic inflammatory disorders, autoimmune diseases, graft-vs.-host disease and allograft rejection (1-3). MSCs are widely distributed in vivo and are found in the bone marrow, adipose tissue, brain, heart, skin, umbilical cord and peripheral blood (4). These cells can be differentiated into numerous tissue phenotypes, including neural, pancreatic and hepatocytic phenotypes, in specific microenvironments (5). The lack or low expression of co-stimulatory factors, such as CD80, CD86, CD40, CD34 and human leukocyte antigen-II, largely benefit the MSC-based therapy (6). Another advantage of MSCs in clinical therapy is their immunosuppressive effect. MSCs are able to inhibit the immune responses by blocking the functions of T-, B-, natural killer (NK) and dendritic cells (7-9).

The mechanism underlying the effect of MSCs on the in vivo biological functions remains unknown (10). In spite of the known immunosuppressive effect of MSCs, several studies have reported that MSCs induced immune responses, resulting in the injury and rejection of MSCs, and eventually leading to the failure of clinical therapy (11-15). Implanted MSCs are recognized by the immune system of recipients and are eliminated (12). Previous studies have confirmed this notion, since MSCs activated NK cell-mediated immune rejection and induced memory $\mathrm{T}$ cell response $(13,14)$. Evidence also indicated the injury of MSCs was mediated by the complement system (15).

Toll-like receptors (TLRs) are a family of pathogen-associated molecular patterns, which serve important roles in the activation of immune responses. There are 11 members in the human TLRs family, which bind to distinct components from 
bacteria, viruses, fungi, as well as circulating endogenous ligands (16). TLR6 is located on the surface of cell membrane and recognizes diacylated lipoprotein of bacteria and conserved regions of dengue virus (17). However, to the best of our knowledge, no previous study has elucidated the role of TLR6 in regulating the immune status of MSCs isolated from the umbilical cord. In the present study, the TLR6 pathway was stimulated by a TLR6 agonist, namely macrophage-activated lipopeptide-2 (MALP-2), and the effects of TLR6 activation on the immune status of umbilical cord MSCs (UCMSCs) was investigated.

\section{Materials and methods}

Culture and stimulation of UCMSCs. Human UCMSCs were provided by the Sichuan Umbilical Cord Blood Stem Cell Bank (Chengdu, China). The UCMSCs were isolated by the cell bank according to a previously described method (18). Briefly, following disinfection in $75 \%$ ethanol for 10-20 min, the umbilical cord was diced and then placed into $25 \mathrm{~mm}^{2}$ plates. The culture medium was added when tissues were firmly attached to the plates (usually 15-20 h). Next, the mesenchymal tissues were removed 5-8 days later, and then dissociated MSCs were collected. The UCMSCs were maintained in Dulbecco's modified Eagle's medium (DMEM; Invitrogen; Thermo Fisher Scientific, Inc., Waltham, MA, USA) supplemented with $10 \%$ fetal bovine serum (Invitrogen; Thermo Fisher Scientific, Inc.) and then stored in liquid nitrogen $\left(-160^{\circ} \mathrm{C}\right)$ for future use. The present study was approved by the Ethics Committee of the West China Second Hospital, Sichuan University (Chengdu, China). The TLR6 agonist MALP-2 was purchased from Novus Biologicals LLC (Littleton, CO, USA) and prepared as recommended by the instruction manual.

Leukocyte proliferation and cell injury assay. Peripheral blood leukocytes (PBLs) were isolated from blood samples of healthy volunteers (subsequent to obtaining written informed consent) by centrifugation gradient $(1,200 \mathrm{x} \mathrm{g}$ for $5 \mathrm{~min}$ and $10^{\circ} \mathrm{C}$ ). PBLs were then labeled with carboxyfluorescein diacetate succinimidyl ester (CFSE) at a final concentration of $10 \mu \mathrm{M}$, and cold complete DMEM was added for $10 \mathrm{~min}$ to stop the reaction. CFSE-labeled PBLs were then washed three times by cold phosphate buffered saline and centrifuged at $1,200 \mathrm{x} \mathrm{g}$ for $5 \mathrm{~min}$ at $10^{\circ} \mathrm{C}$ to remove the unlabeled CFSE. Subsequently, the cells were co-cultured with UCMSCs (co-culture ratio, 10:1) in the presence of MALP-2 $(100 \mu \mathrm{g} / \mathrm{ml})$ and the MALP-2 untreated co-culture group was used as control. UCMSCs treated with 5\% Triton X-100 was used as the positive control, which acted as the maximum release group. Cells were collected at $72 \mathrm{~h}$ after stimulation for detection of the PBL proliferation by fluorescence-activated cell sorting, (increased PBL proliferation dilutes the labeled fluorescence). The supernatants from the two PBL-UCMSC co-culture groups (untreated control and MALP-2-treated group) were collected by centrifugation at 3,000 x $\mathrm{g}$ for $20 \mathrm{~min}$ at $4^{\circ} \mathrm{C}$, and the lactate dehydrogenase (LDH) levels were measured by a cytotoxicity detection kit (Roche Applied Science, Indianapolis, IN, USA) according to the manufacturer's instructions. The lysis percentage was calculated using the following formula: Lysis $(\%)=(\mathrm{E}-\mathrm{M}) /(\mathrm{T}-\mathrm{M}) \times 100$, where
$\mathrm{E}$ is the experimental release, $\mathrm{M}$ is the spontaneous release in the presence of media alone, and $\mathrm{T}$ is the maximum release in the presence of $5 \%$ Triton $\mathrm{X}-100$.

Reverse transcription-quantitative polymerase chain reaction (RT-qPCR) for quantification detection. The mRNA expression levels of interleukin (IL)-1 $\beta$, IL-6, IL-8, IL-9, IL-10, chemokine (C-C motif) ligand 1 (CCL1), CCL2, CCL4, CCL26, Kruppel-like factor 4 (Klf4), Lin28, Nanog, SRY-box 17 (Sox 17) and glyceraldehyde 3-phosphate dehydrogenase (GAPDH) were determined by RT-qPCR. Briefly, total RNA was extracted from the treated and untreated UCMSCs, and reverse transcription was performed using a ReverTra Ace qPCR RT kit (FSQ-101; Toyobo Co., Ltd., Kagoshima, Japan) to synthesize cDNA, using the following conditions: $65^{\circ} \mathrm{C}$ for $5 \mathrm{~min}, 37^{\circ} \mathrm{C}$ for $15 \mathrm{~min}$ and $98^{\circ} \mathrm{C}$ for $5 \mathrm{~min}$. Next, qPCR was conducted with the SYBR Green RealMaster Mix (FP202; Tiangen Biotech Co., Ltd., Beijing, China) under the following thermal cycling conditions: One cycle at $95^{\circ} \mathrm{C}$ for $30 \mathrm{sec}$, 40 cycles at $95^{\circ} \mathrm{C}$ for $30 \mathrm{sec}, 58^{\circ} \mathrm{C}$ for $30 \mathrm{sec}$ and $72^{\circ} \mathrm{C}$ for $30 \mathrm{sec}$, followed by a melt curve from 55 to $95^{\circ} \mathrm{C}$ in $0.5^{\circ} \mathrm{C}$ increments and $10 \mathrm{sec}$ intervals. The primers used for qPCR assay are listed in Table I. GAPDH was used as an internal control. Quantitative data were analyzed using Bio-Rad iQ5 software (Bio-Rad Laboratories, Inc., Hercules, CA, USA). The figures were completed using GraphPad Prism5 (GraphPad Software, Inc., La Jolla, CA, USA).

Detection of surface markers. MALP-2-treated and untreated UCMSCs were harvested at $72 \mathrm{~h}$ post-stimulation. The UCMSCs were stained with different antibodies to detect the expression levels of surface molecules, including co-stimulators and stem cell markers. The antibodies used in this assay were the following: CD40 (cat. no. 11-0809), CD59 (cat. no. 11-0596), CD86 (cat. no. 12-0869) and CD90 (cat. no. 45-0909; all from eBioscience, Thermo Fisher Scientific, Inc.), all were diluted to 1:200 with serum-free DMEM. Flow cytometry was then conducted and analyzed using CXP flow cytometry software (Beckman Coulter, Brea, CA, USA). Positive expression was gated according to the fluorescence intensity of the negative control group.

UCMSC differentiation. UCMSCs were seeded into 6-well plate with a density of $1.5 \times 10^{5}$ cells/well. Conditioned mediums for chondrocyte induction (A10071-01; Gibco; Thermo Fisher Scientific, Inc.), adipocyte induction (A10070-01; Gibco; Thermo Fisher Scientific, Inc.) and osteocyte induction (A10072-01; Gibco; Thermo Fisher Scientific, Inc.) were added in each well with or without MALP-2 treatment. The results of differentiation were collected at 10- and 15-days post-induction, and analyzed using oil-red staining for adipocytes, alizarin red staining for osteocytes and safranin staining for chondrocytes (eBioscience; Thermo Fisher Scientific, Inc.).

Statistical analysis. Values are expressed as the mean \pm standard error of the mean using SPSS version 16.0 software (SPSS, Inc., Chicago, IL, USA). Significant differences of cytotoxicity percentage between MALP-2 treated and untreated groups were analyzed using a Student's t-test. Significant differences 
Table I. List of oligonucleotides used in quantitative polymerase chain reaction analysis.

\begin{tabular}{|c|c|c|c|}
\hline Gene & Forward primer $\left(5^{\prime}-3^{\prime}\right)$ & Reverse primer $\left(5^{\prime}-3^{\prime}\right)$ & GenBank no. \\
\hline IL-1 $\beta$ & ACGAATCTCCGACCACCACT & CCATGGCCACAACAACTGAC & M15330 \\
\hline IL-6 & GACCCAACCACAAATGCCA & GTCATGTCCTGCAGCCACTG & M14584 \\
\hline IL-8 & CTGGCCGTGGCTCTCTTG & CCTTGGCAAAACTGCACCTT & NM_000584 \\
\hline IL-9 & СTCTGTTTGGGCATTCCCTCT & GGGTATCTTGTTTGCATGGTGG & M30134 \\
\hline IL-10 & GGTGATGCCCCAAGCTGA & TCCCCCAGGGAGTTCACA & U16720 \\
\hline CCL1 & GCAGATCATCACCACAGCC & GTCCACATCTTCCGGCCA & NM_002981 \\
\hline CCL2 & CTGCTCTCCAGCGCTCTCA & GTAAGAAAAGCAGCAGGCGG & NM_002984 \\
\hline CCL4 & CAGTGCTTCTGTGCCTGCTG & TGCATCTGGCTGAGCAAGTC & NM_005408 \\
\hline CCL26 & CCAAGACCTGCTGCTTCCAA & GAATTCATAGCTTCGCACCCA & NM_006072 \\
\hline Klf4 & GTCATCAGCGTCAGCAAAGG & CCCTGCTGCTCAGCACTT & NM_004235 \\
\hline Lin28 & TGCTGTCGAGGGATGGATA & CCACCCAATGCGTTCTATTG & NM_024674 \\
\hline Nanog & CCAAAGGCAAACAACCCACTT & CGGGACCTTGTCTTCCTTTTT & NM_001297 \\
\hline Sox 17 & TGGCGCAGCAGAATCCA & CGACTTGCCCAGCATCTTG & NM_022454 \\
\hline GAPDH & GAAGGTGAAGGTCGGAGTC & GAAGATGGTGATGGGATTTC & J04038 \\
\hline
\end{tabular}

MALP-2, macrophage-activated lipopeptide-2; IL, interleukin; CCL, chemokine (C-C motif) ligand; Klf4, Kruppel-like factor 4; Sox 17, SRY-box 17; GAPDH, glyceraldehyde 3-phosphate dehydrogenase.

between untreated UCMSCs and different time points of MALP-2 treated UCMSCs were analyzed by analysis of variance and post hoc test (Tukey's) after homogeneity test of variances. Values of $\mathrm{P}<0.05$ and $\mathrm{P}<0.01$ were considered to indicate statistically significant differences compared with the control group.

\section{Results}

Activation of TLR6 in UCMSCs enhances the proliferation of PBLs. The proliferation of CFSE-labeled PBLs is widely used to detect an increased immune attack. In the present study, CFSE-labeled PBLs were co-cultured with UCMSCs in the presence of MALP-2. PBLs were harvested at 72-h after the co-culture, and the proliferation of PBLs was then detected by flow cytometry. The results indicated that the proliferation of PBLs in the group treated with MALP-2 was increased (18.4\%) as compared with that in the untreated co-culture group (9.2\%), which suggested that TLR6 activation was able to increase the immune response of PBLs against UCMSCs (Fig. 1A).

The damage of cells is frequently detected on the basis of LDH release from injured cells into the culture supernatant. The results of the current study indicated that cytotoxicity percentage were not significantly different between the MALP-2 treated and control groups following 24 and 48 h of co-culture (Fig. 1B). However, the cytotoxicity percentage was significantly increased in the TLR6-activated group compared with the untreated group after $72 \mathrm{~h}(\mathrm{P}<0.05$; Fig. 1B). These results suggested that the activation of TLR6 stimulated the immune attack of PBLs against UCMSCs.

TLR6 activation increases the expression of surface co-stimulatory molecules in UCMSCs. Given the aforementioned results stating that TLR6 activation increased the proliferation and immune attack in the co-culture of PBLs and UMCSCs, the present study also investigated whether MALP-2 treatment influences the expression of surface co-stimulatory factors and stem cell surface markers on UCMSCs. The UCMSCs were treated in the presence or absence of MALP-2, and the surface markers were measured after 72-h incubation by flow cytometry, including measurement of the stimulatory molecules CD40 and CD86, as well as of the stem cell specific markers CD59 and CD90. The results indicated that TLR6 activation enhanced the expression levels of CD40 (15 vs. 4.3\%) and CD86 (13.5 vs. $2.0 \%$ ) as compared with the untreated group (Fig. 2). By contrast, the surface stem cell marker CD90 presented no evident variation, while the expression of CD59 was slightly inhibited in MALP-2 treated UCMSCs (97.9 vs. 90.2\%; Fig. 2).

Treatment with MALP-2 promotes the expression of cytokines and chemokines. RT-qPCR was conducted to detect the expression levels of important cytokines, chemokines and stem cell markers. UCMSCs were stimulated with MALP-2, and total RNA was extracted at 4, 12, 24, 72 and $120 \mathrm{~h}$ post stimulation. qPCR was performed to assay the expression of these important immune system-associated molecules. In cytokine detection, the expression level of IL-10 was significantly induced after treatment for 12-120 h (all $\mathrm{P}<0.01$; Fig. 3A), while IL-1 $\beta$ expression only increased after 24-120 h (24 h, $\mathrm{P}<0.01$; 72-120 h, $\mathrm{P}<0.05)$. IL-6 (12 h, $\mathrm{P}<0.05$; 24-120 h, $\mathrm{P}<0.01$ ) and IL-8 (all $\mathrm{P}<0.01)$ expression increased 12-120 h post stimulation. In chemokine assessment, CCL4 was significantly induced from 24-120 h of treatment (all $\mathrm{P}<0.01$; Fig. 3B), and CCL1 expression increased after $120 \mathrm{~h}$ of stimulation $(\mathrm{P}<0.05)$. However, the other two chemokines, CCL26 (72 h, P<0.05; $120 \mathrm{~h}, \mathrm{P}<0.01)$ and CCL2 (12-24 h, $\mathrm{P}<0.05 ; 72 \mathrm{~h}, \mathrm{P}<0.01 ; 120 \mathrm{~h}, \mathrm{P}<0.05)$, presented inhibited expression, possibly due to the complex biological functions of chemokines in vivo. 

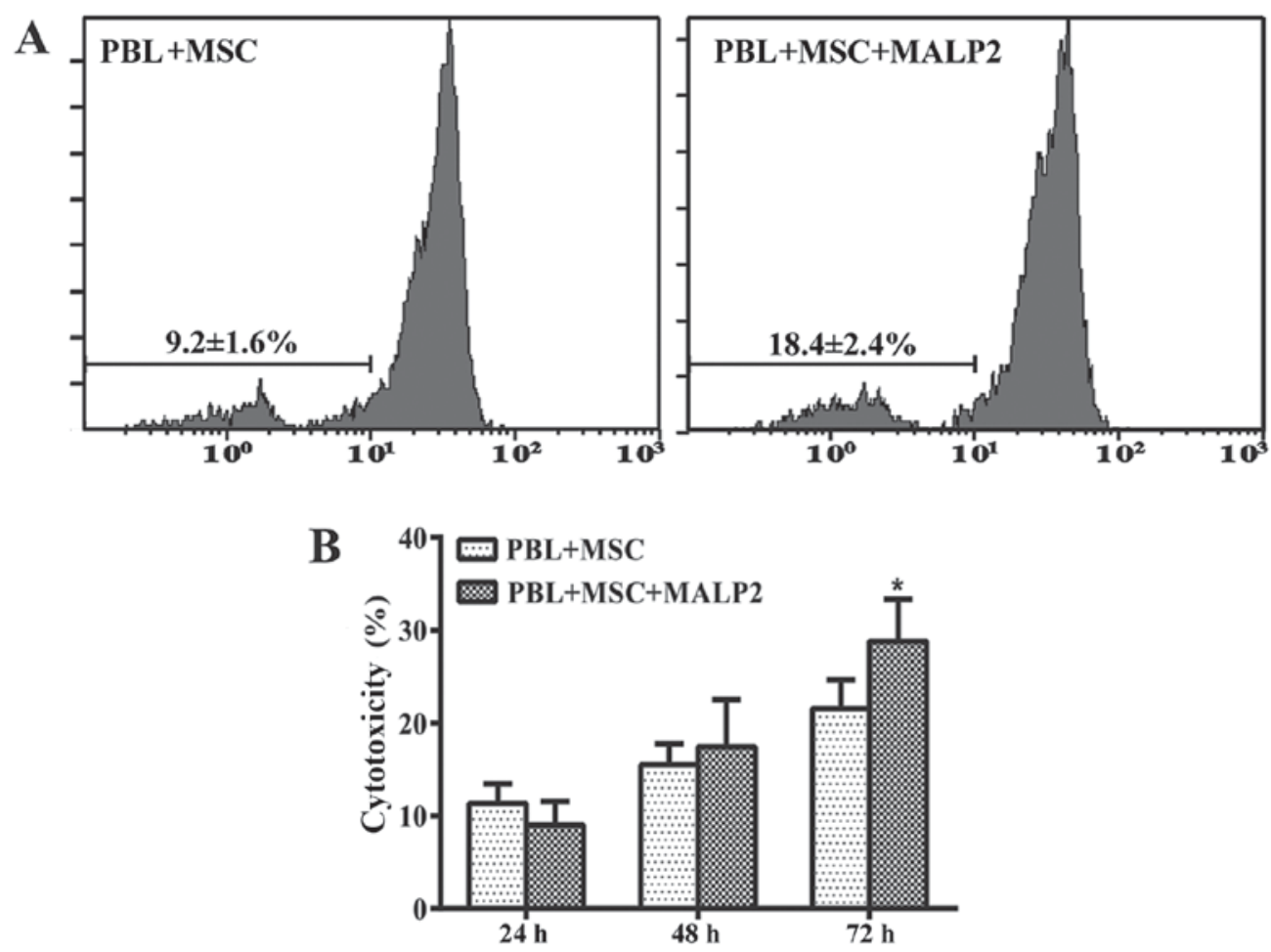

Figure 1. (A) Flow cytometry results on PBL proliferation and (B) detection of LDH level as an indicator of cytotoxicity in the PBL-UCMSC co-culture system. ${ }^{*} \mathrm{P}<0.05$ vs. control group. PBL, peripheral blood leukocyte; MSC, mesenchymal stem cell; UCMSC, umbilical cord MSC; MALP-2, macrophage-activated lipopeptide-2.
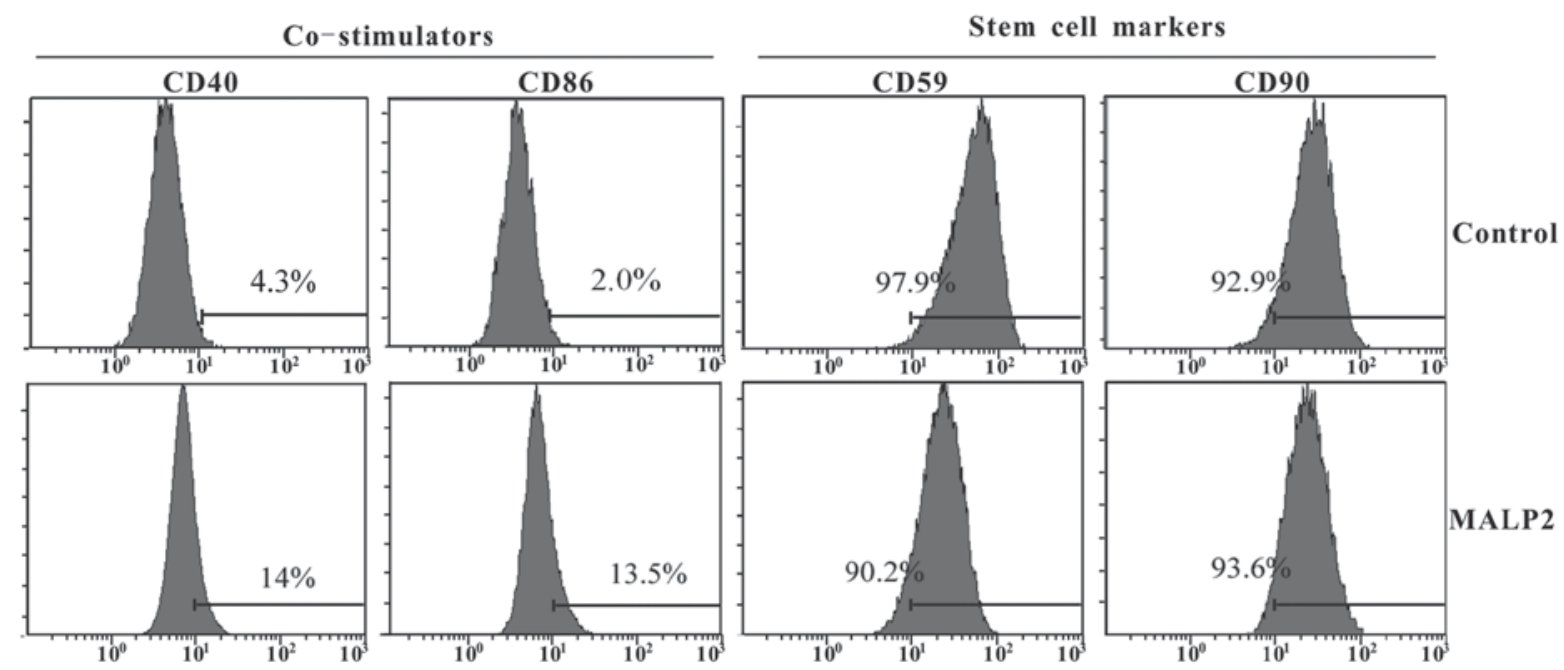

Figure 2. Analysis of co-stimulatory molecules and stem cell markers in UCMSCs upon MALP-2 stimulation. UCMSCs, umbilical cord mesenchymal stem cells; MALP-2, macrophage-activated lipopeptide-2.

To confirm whether activation of TLR6 influences the stemness of UCMSCs, expression levels of stem cell markers, including Klf4, Lin28, Nanog and Sox17, were detected in the present study. The Nanog expression significantly decreased from $4-120 \mathrm{~h}$ of treatment (all $\mathrm{P}<0.01$; Fig. 3C), while Klf4 expression increased at $4 \mathrm{~h}(\mathrm{P}<0.01)$, but was then significantly inhibited from 24-120 h of stimulation with MALP-2 (all P $<0.01$; Fig. 3C). Furthermore, the expression of Lin28 was significantly increased after $12 \mathrm{~h}$ of MALP-2 stimulation $(12 \mathrm{~h}, \mathrm{P}<0.05$; 24-120 h, $\mathrm{P}<0.01$ ), and Sox17 expression was only induced after $120 \mathrm{~h}$ of treatment with MALP-2 (P<0.05; Fig. 3C).
Stimulation with a TLR6 agonist has no effect on the differentiation ability of UCMSCs. The differentiation ability of UCMSCs was also assayed to confirm whether it was regulated by the activation of the TLR6 pathway. Conditional media for adipocytes, osteoblasts and chondrocytes were used to induce UCMSC differentiation. The specific cell phenotypes were identified by different staining methods, more specifically alizarin red for osteoblasts (Fig. 4A), safranin for chondrocytes (Fig. 4B) and oil red $\mathrm{O}$ for adipocytes (Fig. 4C), in order to detect the differentiation status of UCMSCs at 7 and 15 days post MALP-2 treatment. The results indicated that there 

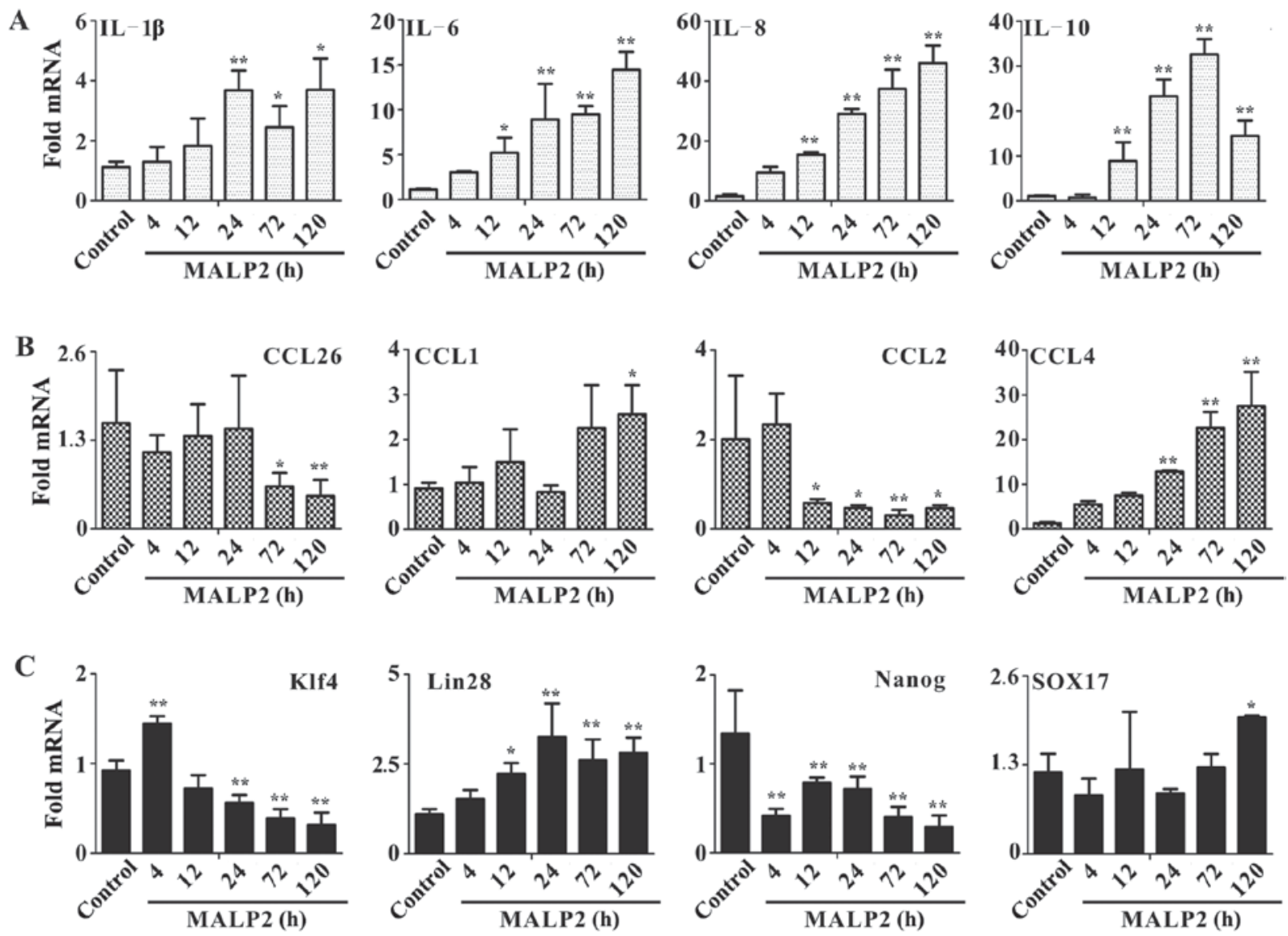

Figure 3. Effect of MALP-2 treatment on the expression levels of (A) proinflammatory cytokines, (B) chemokines and (C) stem cell markers. Data are presented as the mean \pm standard derivation from three independent experiments. ${ }^{*} \mathrm{P}<0.05$ and ${ }^{* *} \mathrm{P}<0.01$ vs. control group. MALP-2, macrophage-activated lipopeptide-2; IL, interleukin; CCL, chemokine (C-C motif) ligand; Klf4, Kruppel-like factor 4; Sox 17, SRY-box 17.

A

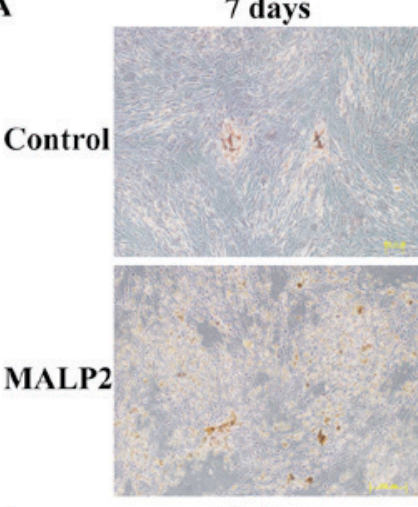

C

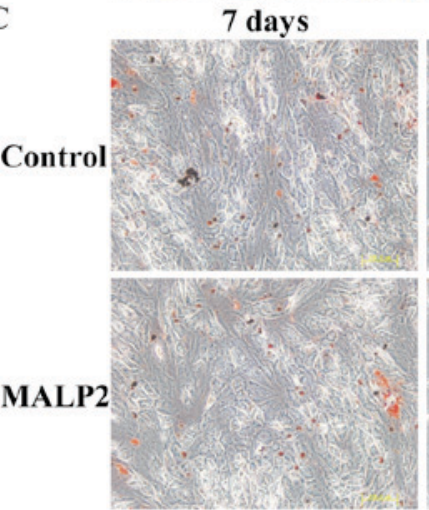

15 days

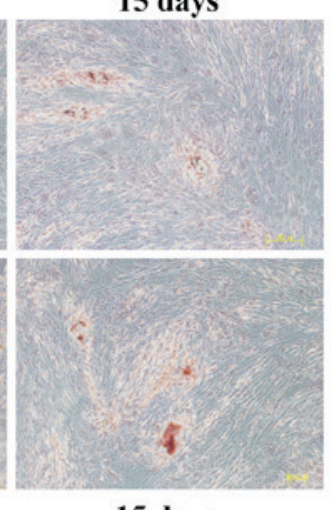

15 days
B

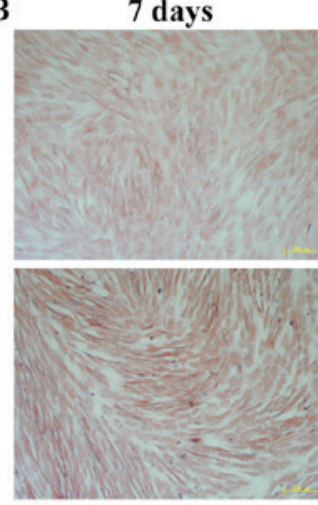

15 days

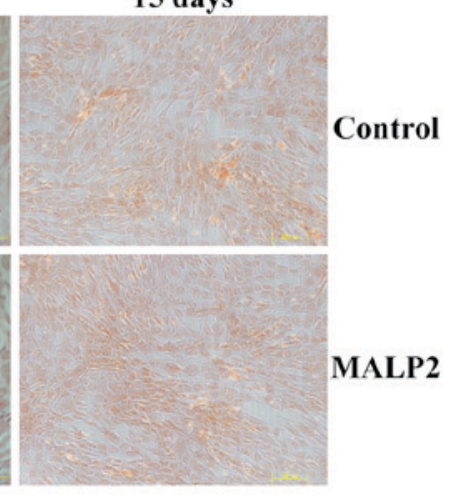

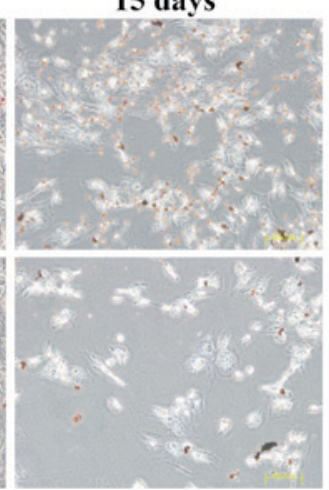

Figure 4. Differentiation of UCMSCs with or without MALP-2 stimulation. Differentiation of (A) osteoblasts, (B) chondrocytes, and (C) adipocytes. UCMSCs, umbilical cord mesenchymal stem cells; MALP-2, macrophage-activated lipopeptide-2. 
were no differences between the control and TLR6-activated groups, which suggested that the TLR6 pathway had no role in regulating the differentiation of UCMSCs (Fig. 4A-C).

\section{Discussion}

The characteristics of MSCs that result in their important role in cell-based therapy include multipotent differentiation ability, lack of co-stimulatory molecule expression and an immunosuppressive effect on the immune system (19). However, recent clinical trials, including those using MSCs to treat graft versus host disease (GVHD), myocardial infarction and liver cirrhosis have not achieved encouraging results due to incomplete understanding of the engrafted MSCs $(11,12)$. Human and animal studies have demonstrated the recognition and elimination of the MSCs within a few days of infusion by modifying the low immune status of MSCs in specific in vivo microenvironments, which eventually resulted in enhanced immune responses.

Proteins of the TLR family are important in bridging innate and adaptive immune response by recognizing conserved components of invasive pathogens, as well as circulating endogenous ligands (20). As UCMSCs is currently becoming more important in cell therapy, the role of TLRs in regulating the biological functions of UCMSCs is critical for improving the controlled and desirable clinical outcomes. Previously, it was confirmed that TLRs are important in regulating the differentiation capacity and immunosuppressive effect, as well as serving a role in MSC survival (21). However, the role of TLRs in the alteration of immunogenicity has seldom been reported. For instance, the study by Zhang et al reported that a TLR7 agonist increased the immunogenicity of UCMSCs (22), while the activation of TLR3, TLR4 and TLR9 had no effect on the immune status of UCMSCs $(23,24)$.

In the present study, the role of TLR6 in the immune status of UCMSCs was assessed by stimulation with the TLR6 agonist, MALP-2. The results indicated that activation of TLR6 increased the proliferation of PBLs and enhanced the release of LDH in the co-culture system, which demonstrated the existence of immune response against UCMSCs. In addition, surface molecule detection revealed increased expression of CD40 and CD86 following stimulation, which are well known co-stimulatory factors in mediating immune response. Furthermore, the expression levels of proinflammatory cytokines (IL-1 $\beta$, IL-6, IL-8 and IL-10) were significantly induced upon MALP-2 stimulation, as well as the levels of two chemokines (CCL1 and CCL4). Huang et al has suggested that differentiation of MSCs into cardiomyocytes increased the immunogenicity of MSCs (25), while Zhang et al also confirmed that activation of TLR7 increased the immune status of UCMSCs by enhancing their differentiation ability (22). Thus, the differentiation capacity of UCMSCs was detected by adding conditional media in the present study, and the results revealed no marked difference between the control and TLR6 agonist-stimulated groups, which suggested that TLR6 had no effect on the differentiation of UCMSCs.

In conclusion, the present study demonstrated that activation of TLR6 resulted in alteration of the immune status of UCMSCs. In clinical trials, the infused UCMSCs will encounter a number of endogenous ligands that may function as agonists to activate different TLR pathways. Thus, future studies are required to confirm the mechanisms underlying the effect of immune response-associated factors in regulating the biological functions of UCMSCs via the TLR6 pathway.

\section{References}

1. Le Blanc K, Frassoni F, Ball L, Locatelli F, Roelofs H, Lewis I, Lanino E, Sundberg B, Bernardo ME, Remberger M, et al: Mesenchymal stem cells for treatment of steroid-resistant, severe, acute graft-versus-host disease: A phase II study. Lancet 371: 1579-1586, 2008

2. Vojtassak J, Danisovic L, Kubes M, Bakos D, Jarabek L, Ulicna $\mathrm{M}$ and Blasko M: Autologous biograft and mesenchymal stem cells in treatment of the diabetic foot. Neuro Endocrinol Lett 27 (Suppl 2): S134-S137, 2006.

3. Guiducci S, Porta F, Saccardi R, Guidi S, Ibba-Manneschi L, Manetti M, Mazzanti B, DalPozzo S, Milia AF, Bellando-Randone S, et al: Autologus mesenchymal stem cells foster revascularization of ischemic limbs in systemic sclerosis: A case report. Ann Intern Med 153: 650-654, 2010.

4. Bordignon C, Carlo-Stella C, Colombo MP, De Vincentiis A, Lanata L, Lemoli RM, Locatelli F, Olivieri A, Rondelli D, Zanon P and Tura S: Cell therapy: Achievements and perspectives. Haematologica 84: 1110-1149, 2011.

5. Phinney DG and Prockop DJ: Concise review: Mesenchymal stem/multipotent stromal cells: The state of transdifferentiation and modes of tissue repair-current views. Stem Cells 25: 2896-2902, 2007.

6. van Poll D, Parekkadan B, Cho CH, Berthiaume F, Nahmias Y, Tilles AW and Yarmuch ML: Mesenchymal stem cell-derived molecules directly modulate hepatocellular death and regeneration in vitro and in vivo. Hepatology 47: 1634-1643, 2008.

7. Shi M, Liu ZW and Wang FS: Immunomodulatory properties and therapeutic application of mesenchymal stem cells. Clin Exp Immunol 164: 1-8, 2011.

8. Han KH, Ro H, Hong JH, Lee EM, Cho B, Yeom HJ, Kim MG, Oh KH, Ahn C and Yang J: Immunosuppressive mechanisms of embryonic stem cells and mesenchymal stem cells in alloimmune response. Transpl Immunol 25: 7-15, 2011.

9. Bassi E, Aita CA and Câmara NO: Immune regulatory properties of multipotent mesenchymal stromal cells: Where do we stand? World J Stem Cells 3: 1-8, 2011.

10. Cui J, Wahl RL, Shen T, Fisher SJ, Recker E, Ginsburg D and Long MW: Bone marrow cell trafficking following intraveneous administration. Br J Haematol 107: 895-902, 1999.

11. Ankrum J and Karp JM: Mesenchymal stem cell therapy: Two steps forward, one step back. Trends Mol Med 16: 203-209, 2010.

12. Allison M: Genzyme back Osiris, despite Prochymal flop. Nat Biotechnol 27: 966-967, 2009.

13. Spaggiari GM, Capobianco A, Becchetti S, Mingari MC and Moretta L: Mesenchymal stem cell-natural killer cell interactions: Evidence that activated NK cells are capable of killing MSCs, whereas MSCs can inhibit IL-2-induced NK-cell proliferation. Blood 107: 1484-1490, 2006.

14. Nauta AJ, Westerhuis G, Kruisselbrink AB, Lurvink EG, Willemze R and Fibbe WE: Donor-derived mesenchymal stem cells are immunogenic in an allogeneic host and stimulate donor graft rejection in a nonmyeloablative setting. Blood 108: 2114-2120, 2006

15. Li Y and Lin F: Mesenchymal stem cells are injured by complement after their contact with serum. Blood 120: 3436-3443, 2012.

16. Blasius AL and Beutler B: Intracellular toll-like receptors. Immunity 32: 305-315, 2010.

17. Chen JC, Ng MM and Chu JJ: Activation of TLR2 and TLR6 by Dengue NS1 protein and its implications in the immunopathogenesis of Dengue virus infection. PLoS Pathog 11: e1005053, 2015.

18. Fu YS, Cheng YC, Lin MY, Cheng H, Chu PM, Chou SC, Shih YH, Ko MH and Sung MS: Conversion of human umbilical cord mesenchymal stem cells in Wharton's jelly to dopaminergic neurons in vitro: Potential therapeutic application for parkinsonism. Stem Cells 24: 115-124, 2006.

19. Tolar J, Le Blanc K, Keating A and Blazar BR: Concise review: Hitting the right spot with mesenchymal stromal cells. Stem Cells 28: 1446-1455, 2011.

20. Akira S, Uematsu S and Takeuchi O: Pathogen recognition and innate immunity. Cell 124: 783-801, 2006. 
21. DelaRosa O and Lombardo E: Modulation of adult mesenchymal stem cells activity by toll-like receptors: Implications on therapeutic potential. Mediators Inflamm 2010: 865601, 2010.

22. Zhang L, Liu D, Pu D, Wang YW, Li L, He YQ, Li YL, Li L and Li WM: The TLR7 agonist Imiquimod promote the immunogenicity of mesenchymal stem cessl. Biol Res 48: 6, 2015.

23. Zhang L, Liu D, Pu D, Wang YW, Li L, He Y, Li YL, Li L, Qiu ZC, Zhao S and Li WM: The role of Toll-like receptor 3 and 4 in regulating the function of mesenchymal stem cells isolated from umbilical cord. Int J Mol Med 35: 1003-1010, 2015.
24. Yang Y, Wang Y, Li L, Bao J, Chen F and Zhang L: Toll-like receptor 9 agonist stimulation enables osteogenic differentiation without altering the immune status of umbilical cord mesenchymal stem cells. Mol Med Rep 12: 8077-8084, 2015.

25. Huang XP, Sun Z, Miyagi Y, McDonald Kinkaid H, Zhang L, Weisel RD and Li RK: Differentiation of allogeneic mesenchymal stem cells induces immunogenicity and limits their long-term benefits for myocardial repair. Circulation 122: 2419-2429, 2010. 